\title{
Moon shadow in ANTARES
}

Tommaso Chiarusi ${ }^{1, *}$ and Matteo Sanguineti ${ }^{2, * *}$ on behalf of the ANTARES Collaboration

${ }^{1}$ INFN - Sezione di Bologna, Viale Berti-Pichat 6/2 40127 Bologna, Italy

${ }^{2}$ INFN - Sezione di Genova, Via Dodecaneso 33, 16146 Genova, Italy

\begin{abstract}
The ANTARES neutrino telescope is the largest Cherenkov detector currently in operation in Mediterranean sea. The search for point-like neutrino sources is one of the telescope goals. For this reason, both the detector angular resolution and the pointing accuracy need a proper estimation. Measuring the deficit of the atmospheric muons in the direction of the Moon induced by the absorption of primary cosmic rays, the so called Moon shadow, allows to evaluate the pointing performance of the telescope. In this contribution we show the result of the analysis of the data taken between 2007 and 2016. The Moon shadow is detected with $3.5 \sigma$ significance. This is the first measurement of the ANTARES angular resolution for atmospheric muons and its absolute pointing using a celestial calibration source. The presented results confirm the good pointing performance of the detector as well as the expected angular resolution.
\end{abstract}

\section{Introduction}

The ANTARES detector (Astronomy with a Neutrino Telescope and Abyss environmental RESearch) [1] currently is the largest submarine neutrino telescope, operating since 2007 in the Mediterranean sea at $2500 \mathrm{~m}$ depth, about $50 \mathrm{~km}$ off the coast of Toulon, in France. It aims to measure the fluxes of cosmic neutrinos arriving either diffuse from the whole sky as well as from potential astrophysical sources. The secondary muons from high energy ( $>10 \mathrm{TeV})$ neutrino interactions in the proximity of the detector are optimal proxies for the impinging progenitors. The goodness of the muon track reconstruction strongly determines the quality of the inferences about neutrino direction and their possible origin. The discovery potential for point-sources finally relies on the absolute orientation of the telescope and its angular resolution.

This contribution summarises the estimations of the pointing performance of the ANTARES detector exploiting the so called Moon shadow effect: the deficit in the flux of atmospheric muons coming from the direction of the Moon disk due to the absorption of the primary cosmic rays by the Moon itself. The detailed description of all the analysis procedures can be found in [2].

\section{Optimisation of the selection criteria}

The quality cuts used in the present analysis relate to the two main parameters from the reconstruction of the muon tracks at the detector level: the error on the reconstructed muon

\footnotetext{
*e-mail: tommaso.chiarusi@bo.infn.it

**e-mail: matteo.sanguineti@ge.infn.it
} 
direction and a likelihood based estimator of the linear track fit, described in [3]. The cut optimization is based on dedicated Monte Carlo (MC) simulation of atmospheric muons with the MUPAGE code [4]. The full MC chain comprises the generation of muons at the detector level coming from the upper $2 \pi$ sky, the production and detection of the Cherenkov light emitted along the muon tracks, including occasional optical background from bioluminescence and ${ }^{40} \mathrm{~K}$ decays. The algorithms for track reconstruction are the same ones used also for the real data. The MC simulations are performed considering the real conditions of the ANTARES data taking, evolving along with the detector operations from 2007 to 2016 [5]. Only the event tracks reconstructed as down-going and detected when the Moon is above the horizon are selected. For this analysis two different sets of Monte Carlo simulation are

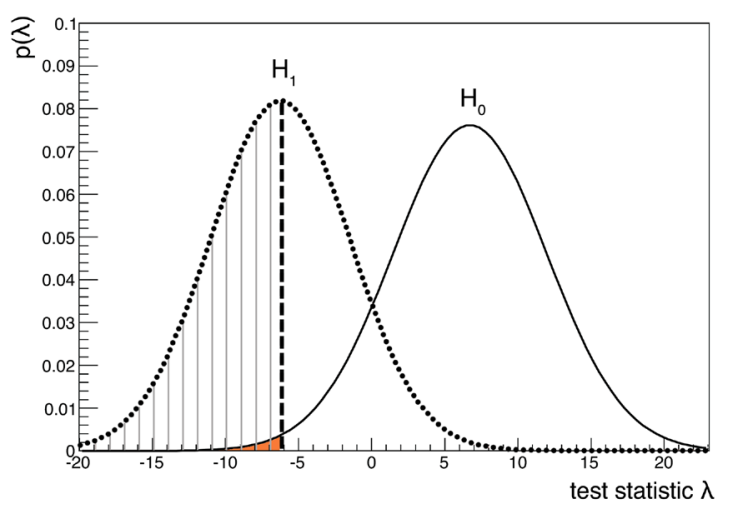

Figure 1. The distribution of the test statistic $\lambda$ from eq. 1 for the "no Moon shadow" hypothesis $H_{0}$ (full curve) and the "Moon shadow" hypothesis $H_{1}$ (dotted curve). The dashed area corresponds to the $50 \%$ of the pseudo-experiments where the Moon shadow hypothesis $H_{1}$ is correctly identified. The tiny shaded area quantifies the expected median significance of $3.4 \sigma$ to observe the Moon shadow.

realised: the first one without considering any effect due to the Moon $\left(H_{0}\right.$ or null hypothesis); the second one with the shadowing effect ( $H_{1}$ hypothesis), implemented by rejecting the muons generated within the Moon disk of angular radius $R_{\text {Moon }}=0.26^{\circ}$.

The event density distribution as function of the angular distance from the nominal position of the Moon is determined, for both the MC sets, in a field of view (FoV) of $10^{\circ}$ radius and subdivided in circular equal-space bins, $0.4^{\circ}$ wide. The content $N_{i, H}$ of the $i$-th bin under a given hypothesis $H$ is taken as the expectation value of the related occurrences $n_{i}$ obtained in $10^{6}$ pseudo-experiments with a certain value of the quality cuts. It is then possible to determine the distribution of the test-statistic $\lambda$, as expressed by equation (1), for each scenario $s$ of null/shadowing hypothesis:

$$
\lambda_{s}=\chi_{H_{0} \mid s}^{2}-\chi_{H_{1} \mid s}^{2}, \quad \text { with } \quad \chi_{H \mid s}^{2}=2 \sum_{i=1}^{N_{\text {bin }}}\left[N_{i, H}-n_{i \mid s}+n_{i} \ln \frac{n_{i \mid s}}{N_{i, H}}\right] .
$$

Figure 1 shows the $\lambda$ distributions $H_{0}$-wise, on the right, and $H_{1}$-wise, on the left, for the obtained optimal cuts applied to the MC sets. From the separation of the two curves it is possible to compute a $3.4 \sigma$ expected significance of the Moon shadowing effect. 


\section{One dimensional data analysis}

Figure 2 shows the measured muon event density as a function of the angular distance from the Moon for the data sample 2007-2016. Assuming a Gaussian shape for the detector point spread function (PSF), it is possible to evaluate the detector angular resolution fitting the event density with the relation:

$$
\frac{d n}{d \delta^{2}}=k\left(1-\frac{R_{\text {Moon }}^{2}}{2 \sigma_{\text {res }}^{2}} e^{-\frac{\delta^{2}}{2 \sigma_{\text {res }}^{2}}}\right)
$$

where $\delta$ is the angular distance from the Moon centre. The two free parameters are $k$, the average muon event density in the $H_{0}$ scenario, and $\sigma_{r e s}$, the angular resolution. The Moon radius $R_{\text {Moon }}$ is fixed to $0.26^{\circ}$. The angular resolution for downward-going atmospheric muons resulting from the fit is $\sigma_{\text {res }}=0.73^{\circ} \pm 0.14^{\circ}$. The significance of the shadowing is evaluated using a $\chi^{2}$ test comparing the measured event density with the flat distribution $\frac{d n}{d \delta^{2}}=k$. Such a $\chi^{2}$ test leads to a significance of the Moon shadow effect of $3.3 \sigma$.

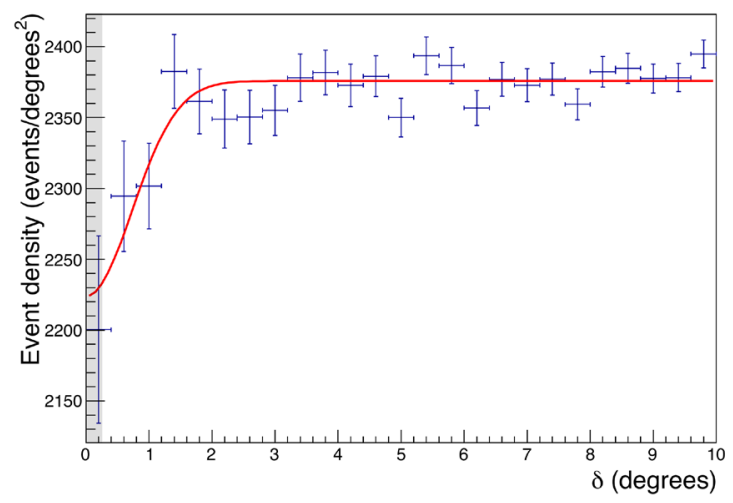

Figure 2. Measured muon event density as a function of the angular distance $\delta$ from the Moon. The smooth line is the best fit according to equation (2); the shaded area corresponds to the apparent radius of the Moon $\left(0.26^{\circ}\right)$.

\section{Two dimensional data analysis}

The ANTARES pointing accuracy is evaluated by comparing the point in the sky where the Moon shadow is found with the largest significance to the nominal position of the Moon. For this study, the $10^{\circ} \times 10^{\circ} \mathrm{FoV}$ around the Moon position is boxed in a grid of $0.2^{\circ} \times 0.2^{\circ}$ squared bins. The used test statistic is again $\lambda$ as described above. This time the scenario is the one with the shadowing effect, with the Moon center recursively tested in each bin of the FoV. For each test, the sum in eq.(1) is evaluated over all the $100 \times 100$ bins in the FoV and the value of the test statistic $\lambda$ is minimised finding the best fit of a bi-dimensional Gaussian PSF (Fig.3, left) overimposed on the due background of atmospheric muons. The smallest value $\lambda_{\min }$ is found equal to -17.05 in the FoV coordinates $\left(0.5^{\circ}, 0.1^{\circ}\right)$ and corresponds to a significance of $3.5 \sigma$ of the Moon shadow effect. Confidence intervals are shown in Fig.3, right, see caption for details. 


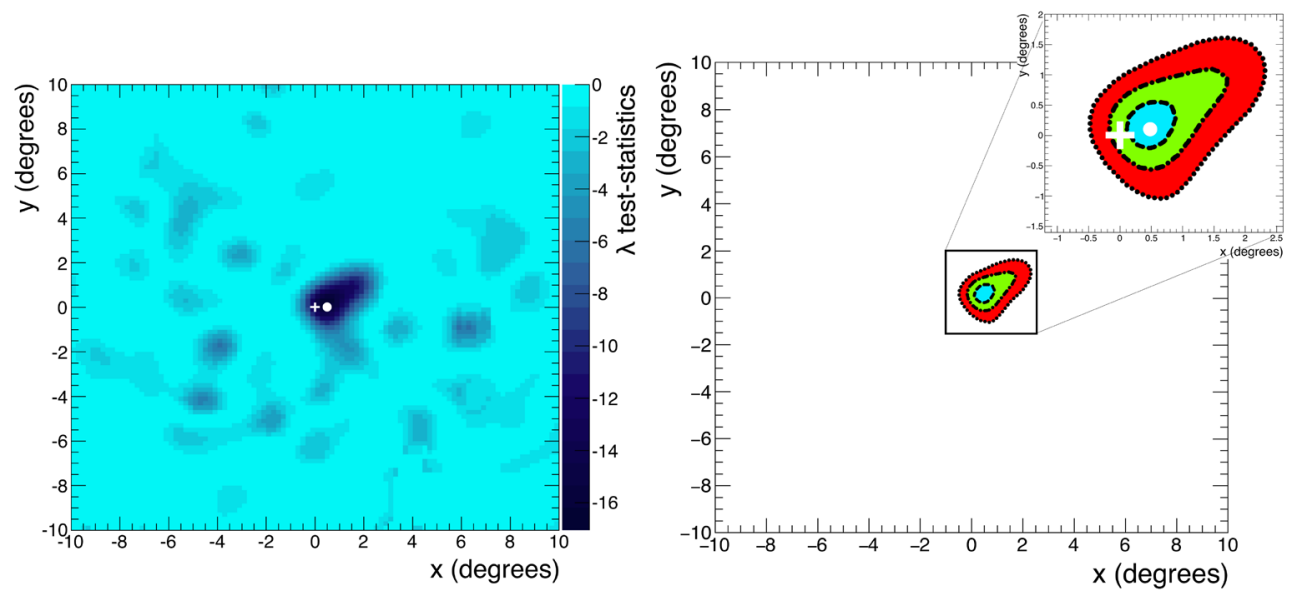

Figure 3. Left: Measured distribution of the test statistic $\lambda$ from equation (1) around the nominal position of the Moon, assumed in the center of the FoV. Right: Contour plots for different confidence levels (cyan/dashed: 68.27\%; green/dot-dashed: $95.45 \%$; red/dotted: $99.73 \%$ ). In both plots, the white dot represents the position of $\lambda_{\min }$ while the cross indicates the nominal position of the Moon. The small discrepancy between the two (1.2 $\sigma$ significance) is compliant with a statistical fluctuation.

\section{Conclusions}

The pointing performance of the ANTARES telescope has been estimated using the Moon shadow effect. The analysis of the ANTARES data sample of the years 2007-2016 allowed the identification of the Moon shadow effect with $3.5 \sigma$ significance and the detector angular resolution for downward going atmospheric muons of $0.73^{\circ} \pm 0.14^{\circ}$. The pointing accuracy of the detector is consistent with the expectations.

\section{References}

[1] M. Ageron et al. (ANTARES), Nucl. Instrum. Meth. A656, 11 (2011), 1104 . 1607

[2] A. Albert et al., The European Physical Journal C 78, 1006 (2018)

[3] S. Adrian-Martinez et al. (ANTARES), JCAP 1303, 006 (2013), 1302 . 6750

[4] G. Carminati, A. Margiotta, M. Spurio, Comput. Phys. Commun. 179, 915 (2008), 0802.0562

[5] Fusco, L.A., Margiotta, A., EPJ Web of Conferences 116, 02002 (2016) 